\title{
Healthy brooders employ more attentional resources when disengaging from the negative: an event-related fMRI study
}

\author{
Marie-Anne Vanderhasselt • Simone Kühn • \\ Rudi De Raedt
}

Published online: 4 March 2011

(C) Psychonomic Society, Inc. 2011

\begin{abstract}
Depressive brooding is considered a maladaptive ruminative-thinking style that has been shown to be highly correlated with major depression. The present study in healthy participants employed event-related fMRI to uncover the neural underpinnings of emotional disengagement as it relates to depressive brooding. Thirty-four healthy, never depressed individuals performed an emotional go/nogo task with a rapid presentation of emotional faces. We focused on the contrast of inhibiting sad (happy/no-go) versus inhibiting happy (sad/no-go) information. This contrast allowed us to assess possible difficulties in disengaging from emotionally negative, as compared with emotionally positive, faces. At the behavioral level, only in high brooders were higher self-reported brooding scores correlated with more errors when sad information was inhibited, relative to happy information. At the neural level, across all participants, brooding scores were positively correlated with activity in the right dorsolateral prefrontal cortex (DLPFC; BA 46), implying that high brooders show higher DLPFC involvement when successfully disengaging from a series of negative stimuli. These results may suggest that healthy individuals who report a high brooding thinking style need to recruit more attentional control in order to disengage successfully from negative information,
\end{abstract}

Marie-Anne Vanderhasselt and Simone Kühn share a first authorship.

M.-A. Vanderhasselt $(\bowtie) \cdot S$. Kühn $\cdot$ R. De Raedt

Department of Experimental and Clinical Health Psychology,

Ghent University,

Henri Dunantlaan 2,

9000 Gent, Belgium

e-mail: MarieAnne.Vanderhasselt@Ugent.be

S. Kühn

Charité University Medicine, Campus Mitte,

St. Hedwig-Krankenhaus, Clinic for Psychiatry and Psychotherapy,

Große Hamburger Straße 5-11,

10115 Berlin, Germany in a way that may be related to emotion regulation strategies. These mechanisms might protect them from developing depressive symptoms.

Keywords Depressive brooding $\cdot$ go/no-go $\cdot$ Emotional disengagement $\cdot$ DLPFC $\cdot$ Emotion regulation

From everyday life experience, we all know that it is sometimes hard to stop thinking about a negative event or stressor. When these thoughts occur more frequently and an individual focuses passively on the experience of negative mood states, this is considered depressive brooding - that is, a particular ruminative-thinking style (Joormann, Dkane, \& Gotlib 2006; Treynor, Gonzalez, \& Nolen-Hoeksema 2003). Brooding is of the utmost clinical importance because, although brooders are not necessarily currently depressed, brooding is a trait that increases vulnerability to depression (Nolan, Roberts, \& Gotlib 1998; Nolen-Hoeksema, 2000; Roberts, Gilboa, \& Gotlib 1998). It is important to note that there are several types of repetitive thought that are related to depression vulnerability (e.g., stress-reactive rumination; see Robinson \& Alloy, 2003). Depressive brooding is a particular type of repetitive thought where negative mood is considered a necessary component for triggering ruminative thought processes (Nolen-Hoeksema, 2000).

Cognitive research focusing on the information processing underlying depressive brooding has flourished over the past decade. Joormann et al., (2006) demonstrated that the tendency to attend to negative information in depression (i.e., negative attentional bias) is specifically related to brooding. More recently, it has been shown that brooding is related to impairments in attentional control processes - the inhibition of previously relevant task sets (Whitmer \& Banich, 2007) and, more specifically, to impairments in inhibiting negative information (De Lissnyder, Koster, Derakshan, \& De Raedt 
2010). Therefore, it is likely that a difficulty in inhibiting and disengaging from negative information is the mechanism underlying brooding (Banich et al., 2009; Hertel, 2007; Joormann, 2004; Koster, De Lissnyder, Derakshan, \& De Raedt 2010). Overall, these attentional deficits in inhibiting and disengaging from negative information appear to be the key mechanism for impairments in the regulation of emotions (Joormann, 2010).

Despite the recent increase in cognitive research on depressive brooding, Banich et al. (2009) have pointed out that the neural underpinnings of these inhibition mechanisms remain to be determined. Most neuroimaging studies on rumination and the sustained processing of negative information have been based on emotional regulation designs (Ray et al., 2005; Siegle, Steinhauer, Thase, Stenger, \& Carter 2002). However, the ability to suppress or reappraise negative thoughts (key functions of emotion regulation) might be related to individual differences in attentional control over negative information (for a review, see De Raedt \& Koster, 2010). Recently, a neuroimaging study by Berman, Peltier, Nee, Kross, Deldin, and Jonides (2011) in depressed patients demonstrated that higher brooding scores were related to an increased connectivity between the posterior and subgenual cingulate cortex during off-task periods and that these findings remained when depressive symptoms were controlled for. These findings demonstrate that depressive brooding is related to specific brain activation patterns, especially when individuals are at rest. Moreover, Berman, Nee, Casement, Kim, Deldin, Kross, Jonides (2011) demonstrated that depressed patients, who show higher depressive brooding scores, show more spatial variability in the activation of the left inferior frontal gyrus (IFG), which might reflect an inability to inhibit negative information. Nevertheless, it remains relatively unclear how interindividual differences in brooding thinking styles are related to neural underpinnings of interindividual differences in attentional control for emotional information. This is important given the abovementioned cognitive research reporting an association between brooding tendencies and attentional control impairments. Moreover, because depressed patients are known to be impaired in attentional control processes, it is especially important to investigate a homogeneous group of individuals reporting no (history of) depressive symptoms. In an effort to close this gap, we have designed a study that implements fMRI during a task in which healthy individuals need to inhibit emotional information. These behavioral and neural correlates will be related to a selfreport measure of depressive brooding.

To this end, we chose a task that challenges participants to focus their attention on a continuous stream of negative stimuli and to inhibit their response to a single positive stimulus. More specifically, we used an emotional variant of the go/no-go task that tests the participant's ability to inhibit a frequent go response during an infrequent no-go trial (Aron, Robbins, \& Poldrack 2004; Schulz et al., 2007). This go/no-go task uses emotionally valenced stimuli to examine inhibition when participants have developed an attentional set for specific emotional information. The attention can be focused onto positive information; participants develop a prepotent response to positive stimuli and need to inhibit this response when confronted with a negative stimulus. On the other hand, the attention can be focused onto negative information; participants develop a prepotent response to negative stimuli and need to inhibit this response when confronted with a positive stimulus. We compared these two manipulations by contrasting the processes of inhibiting negative and inhibiting positive information. By comparing the two no-go conditions, we cancelled out the motor response inhibition component in order to focus solely on the updating of the attentional set to disengage from emotional information.

The emotional go/no-go task therefore serves as an effective paradigm for exploring interindividual differences in emotional disengagement and how they relate to interindividual differences in brooding tendencies. There is a large body of neuroimaging literature that has related attentional processes for emotional and nonemotional information to prefrontal brain activation. In particular, the right dorsolateral prefrontal cortex (DLPFC) has been associated with maintaining task-relevant information online (MacDonald, Cohen, Stenger, \& Carter 2000; Vanderhasselt et al., 2007; for a review, see Vanderhasselt, De Raedt, \& Baeken 2009), the inhibition of negative information during a negative affective priming task (Leyman, De Raedt, Vanderhasselt, \& Baeken 2009), and disengagement from negative information during an exogenous cuing task (De Raedt et al., 2010).

Taken together, on the basis of the literature cited above, one could predict that high brooders will have more difficulty in the emotional go/no-go task-specifically, when disengaging from negative information. This could result in a lower behavioral accuracy and might be related to activation of the DLPFC.

\section{Method}

Participants

Postings on the university Web site were used to recruit a group of 34 participants with a mean age of 21.56 years $(S D=2.35 ; 9 \mathrm{M} / 25 \mathrm{~F})$ and without (a history of) psychopathology or neurological conditions. All participants were right-handed and were eligible for fMRI research. The Dutch version of the Beck Depression 
Inventory (BDI-II; Beck, Steer, \& Brown 1996; Van der Does, 2002) was administered in order to screen for depressive symptoms $(M=5.53, S D=3.95)$.

\section{Procedure}

The study protocol was approved by the local Medical Ethics Committee of Ghent University Hospital. All participants were initially screened for inclusion/exclusion criteria and gave written informed consent prior to the study. Subsequently, the emotional go/no-go task was administered in the fMRI scanner. Finally, participants rated all the experimental faces on valence and arousal and filled in emotion regulation questionnaires. Total participation lasted approximately $2 \mathrm{~h}$, for which they received financial compensation of $\$ 30$.

Emotional go/no-go task The emotional go/no-go task was programmed using E-Prime software (Psychology Software Tools, Pittsburgh, PA). Participants viewed a series of faces (happy and sad), each presented for $500 \mathrm{~ms}$, with an intertrial interval of $1,250 \mathrm{~ms}$. Participants were instructed to look continuously at the center of the screen and to respond (by pressing a button with the thumb of their right hand) as quickly and accurately as possible. Forty-five faces ( 25 female and 20 male actors) from the Karolinska Directed Emotional Faces data set (KDEF; Lundqvist, Flykt, \& Öhman 1998) were used as stimuli. Each face was shown once with a happy and once with a sad expression. The sad and happy faces were matched on arousal on the basis of a validation study of the KDEF picture set (Goeleven, De Raedt, Leyman, \& Verschuere 2008).

This emotional go/no-go paradigm contained two task blocks that differed in emotional context: (1) withhold a response to infrequent positive stimuli (20\% happy/no-go; inhibiting sad information) in the context of responding to frequent negative stimuli $(80 \% \mathrm{sad} / \mathrm{go}$; responding to $\mathrm{sad}$ information) and (2) withhold a response to negative stimuli (20\% sad/no-go; inhibiting happy information) in the context of responding to frequent positive stimuli $(80 \%$ happy/go; responding to happy information). Before each block, participants were informed that, in the next series of faces, most of the faces would contain a specific emotion (based on the block, either happy or sad) but that some faces could also contain the other emotional expression. They were instructed to press as quickly and as accurately as possible in response to the facial expression that appeared most of the time (based on the block, either happy or sad).

Participants initially completed a practice block of 16 trials (using five faces not shown in the experimental blocks, 12 go and 4 no-go trials), followed by four scanning runs, each containing two blocks (one block to inhibit a response to sad faces and one block to inhibit a response to happy faces) of 100 trials ( 80 go and 20 no-go trials). In total, we presented 80 trials where sad information needed to be inhibited (happy/no-go) and 80 trials where happy information needed to be inhibited (sad/no-go). The order of the four blocks was randomized across participants, with a sequential order of the trials within each block. There were never more than two no-go stimuli presented in a row.

Moreover, 20 blank screens (i.e., null event; $500 \mathrm{~ms}$ ) were randomly intermixed in each condition to serve as a baseline. Each block lasted about $8.30 \mathrm{~min}$, and between blocks, participants were allowed to rest for $30 \mathrm{~s}$.

After completing the emotional go/no-go task, all the participants rated the faces for valence and arousal, using 9point Likert scales (valence, $1=$ unhappy, $5=$ neutral, $9=$ happy; arousal, $1=$ calm, $5=$ intermediate, $9=$ excited $)$.

Self-report measures of rumination and emotion regulation In order to assess the tendency for depressive brooding, we administered the Rumination Response Scale (RRS; NolenHoeksema \& Morrow, 1991; Treynor et al., 2003; Dutch translation by Raes \& Hermans, 2007; RRS-NL; Schoofs, Hermans, \& Raes 2011). The RRS can also be used to assess a measure of reflective pondering, which is, as compared with depressive brooding, a more adaptive form of rumination. Reappraisal and suppression (two emotion regulation styles) were assessed by means of the EmotionRegulation Questionnaire (ERQ; Gross \& John, 2003). For all questionnaires, higher scores are indicative of, respectively, more depressive brooding, reflective pondering, depressive symptoms, and relatively more use of a specific type of emotion regulation style.

Scanning procedure Images were collected with a 3T Magnetom Trio MRI scanner system (Siemens Medical Systems, Erlangen, Germany) using an eight-channel radio frequency head coil. First, high-resolution anatomical images were acquired using a T1-weighted 3D MPRAGE sequence $(\mathrm{TR}=2,530 \mathrm{~ms}$, TE $=2.58 \mathrm{~ms}$, TI $=1,100 \mathrm{~ms}$, acquisition matrix $=256 \times 256 \times 176$, sagittal $\mathrm{FOV}=220$ $\mathrm{mm}$, flip angle $=7^{\circ}$, voxel size $=0.86 \times 0.86 \times 0.9 \mathrm{~mm}^{3}$ ). Whole-brain functional images were collected using a T2*-weighted EPI sequence sensitive to BOLD contrast $(\mathrm{TR}=2,000 \mathrm{~ms}, \mathrm{TE}=35 \mathrm{~ms}$, image matrix $=64 \times 64$, $\mathrm{FOV}=224 \mathrm{~mm}$, flip angle $=80^{\circ}$, slice thickness $=3.0 \mathrm{~mm}$, distance factor $=17 \%$, voxel size $3.5 \times 3.5 \times 3 \mathrm{~mm}^{3}, 30$ axial slices). About 250 image volumes aligned to AC-PC were acquired per run.

fMRI data preprocessing and general linear model analysis

The fMRI data were analyzed with statistical parametric mapping using SPM5 software (Wellcome Department of 
Cognitive Neurology, London). The first four volumes of all the EPI series were excluded from the analysis to allow the magnetization to approach a dynamic equilibrium. Data processing started with slice time correction and realignment of the EPI data sets. A mean image for all the EPI volumes was created, to which individual volumes were spatially realigned by rigid body transformations. The highresolution structural image was coregistered with the mean image of the EPI series. The structural image was then normalized to the Montreal Neurological Institute (MNI) template, and the normalization parameters were applied to the EPI images to ensure an anatomically informed normalization. During normalization, the anatomy image volumes were resampled to $1 \times 1 \times 1 \mathrm{~mm}^{3}$. A filter of $8 \mathrm{~mm}$ FWHM (full-width at half maximum) was used. Lowfrequency drifts in the time domain were removed by modeling the time series for each voxel by a set of discrete cosine functions to which a cutoff of $128 \mathrm{~s}$ was applied. The subject-level statistical analyses were performed using the general linear model. The model contained separate regressors for the blocks in which participants responded to happy faces (happy/go) and the blocks in which they responded to sad faces (sad/go). Furthermore, onsets of nogo trials were modeled as a linear combination of zeroduration events of five types, with separate regressors for correct inhibiting happy information (sad/no-go trials), erroneously inhibiting happy information, correctly inhibiting sad information (happy/no-go trials), erroneously inhibiting sad information, and null events. Using this model of our rapid fMRI design, we were unable to look at brain activity during go trials. Movement parameters were included to account for variance associated with head motion. All resulting vectors were convolved with the canonical hemodynamic response function and its temporal derivative to form the main regressors in the design matrix (the regression model). The statistical parameter estimates were computed separately for each voxel for all the columns in the design matrix. Contrast images were constructed for each individual. Next, a group-level random effects analysis was performed. Brooding scores were correlated with brain activity resulting from the contrast inhibiting happy $(\mathrm{sad} /$ no-go) < inhibiting sad (happy/nogo). The resulting statistical values were thresholded with a level of significance of $p<.001$ (uncorrected). To check for multiple comparisons when examining the effects of brooding on BOLD responses, we used a small volume correction in the frontal cortex where brooding effects were hypothesized a priori. The small volume correction was conducted using anatomical masks created on the basis of the WFU Pickatlas (Maldjian, Laurienti, Burdette, \& Kraft 2003). The resulting maps were overlaid onto a normalized T1-weighted MNI template (colin27), and the coordinates reported corresponded to the MNI coordinate system.
Percentages of signal change analysis

In order to extract percentages of signal changes, we used a sphere with a radius of $6 \mathrm{~mm}$ around the peak coordinate of interest. For each participant, for region and condition separately, the mean percentage of signal change over a time window of 4-6 s after stimulus onset was computed (http://marsbar.sourceforge.net/; Brett, Anton, Valabregue, \& Poline 2002).

\section{Results}

The entire statistical analysis was performed using the SPSS software package (version 15.0).

Self-report questionnaire data Table 1 shows self-reports on the RRS (total scores, depressive brooding, and reflective pondering), BDI-II and ERQ (suppression and reappraisal). Participants reported a mean brooding score of $9.71(S D=3.25$; range 5-17). Depressive brooding was correlated with BDI scores, a measure of depressive severity, $r(34)=.56, p=.001$.

Behavioral data Our dependent variables were the percentage of accuracy for no-go trials: (number of correct no-go trials/total number of no-go trials) $* 100$. Table 2 shows the accuracy for the emotional go/no-go task, separated for high and low brooders. Three dependent variables were calculated: (1) accuracy rates for inhibiting a response to sad information (happy/no-go), (2) accuracy rates for inhibiting a response to happy information (sad/no-go), and (3) accuracy rates for disengaging attention from negative information (accuracy rates for inhibiting sad information minus accuracy rate for inhibiting happy information). The smaller this difference score, the more the participants had difficulty disengaging their attention from negative, relative to positive, information.

Across all participants, we observed no correlation between brooding tendencies and (1) accuracy rates of the inhibition of happy information, $r(34)=.01, p=.93$, (2)

Table 1 Mean scores (with standard deviations) of all self-report questionnaires $(N=34)$

\begin{tabular}{lcc}
\hline Questionnaire & $M(S D)$ & Score Range \\
\hline RRS/total scores & $50.74(15.59)$ & $28-87$ \\
RRS/depressive brooding & $9.71(3.25)$ & $5-17$ \\
RRS/reflective pondering & $10.03(3.83)$ & $5-20$ \\
BDI-II & $5.53(3.95)$ & $0-14$ \\
Emotion regulation/reappraisal & $30.03(4.70)$ & $16-39$ \\
Emotion regulation/suppression & $12.73(4.30)$ & $4-20$ \\
\hline
\end{tabular}


Table 2 Mean accuracy rates (in percentages, with standard deviations) of all participants and of two groups based on a median split: high brooders $(n=19 ;[5-9] ; M=7.22, S D=1.44)$ and low brooders $(n=165 ;[10-17] ; M=12.50, S D=2.28)$

\begin{tabular}{llll}
\hline & $\begin{array}{l}\text { Inhibiting Negative Information (Happy/ } \\
\text { No-Go) }\end{array}$ & $\begin{array}{l}\text { Inhibiting Positive Information (Sad/ } \\
\text { No-Go) }\end{array}$ & $\begin{array}{l}\text { Happy/No-Go Minus Sad/ } \\
\text { No-Go }\end{array}$ \\
\hline All participants & $80.15(8.78)$ & $82.57(8.95)$ & $-2.43(4.70)$ \\
$\begin{array}{l}\text { Low depressive } \\
\text { brooders }\end{array}$ & $79.58(9.74)$ & $82.36(10.13)$ & $-2.78(5.48)$ \\
$\begin{array}{l}\text { High depressive } \\
\text { brooders }\end{array}$ & $80.78(7.84)$ & $82.81(7.73)$ & $-2.03(3.76)$ \\
\hline
\end{tabular}

accuracy rates of the inhibition of sad information, $r(34)=.03$, $p=.86$, and (3) the difference between the two accuracy rates, $r(34)=.05, p=.77$ (see Fig. 1).

In addition, we divided our study sample into two groups on the basis of a median split: low brooders $(n=19$; [5-9]; $M=7.22, S D=1.44)$ and high brooders $(n=15$; [10-17]; $M=12.50, S D=2.28$ ). No correlation between brooding tendencies and the accuracy rates was observed in the two groups, $r \mathrm{~s}<.34, p s>.31$. However, we observed a significant correlation between brooding scores and the difference score in accuracy between inhibiting sad information and inhibiting happy information for high brooders, $r(15)=-.50, p<.05$, but not for low brooders, $r(19)=.34$, $p>.05$. These findings indicate that, in high brooders, higher brooding scores are related to a smaller difference score, indicating relatively more errors in disengaging from negative than from positive information.

As a control, we investigated the tendency to use reflective pondering, the more adaptive form of rumination. Mirroring statistical analyses in brooding scores, we analyzed whether reflection was related to accuracy rates for inhibiting happy or sad information or for the difference score between the two. We found no significant correlation across all participants for these three measures, $p \mathrm{~s}>.31$. Furthermore, no correlation was found when the two

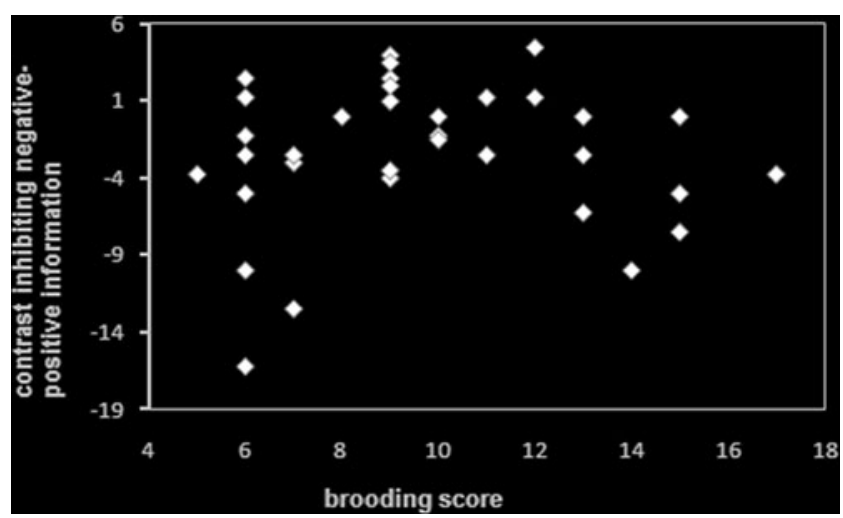

Fig. 1 Accuracy rates for the contrast inhibiting negative minus positive information (accuracy rates for happy/no-go minus sad/nogo). Across all participants, no correlation was observed between brooding scores and accuracy rates, $r(34)=.05, p=.77$ groups were divided into low reflectors ( $n=18$; [5-10]; $M=7.11, S D=1.64), p s>.55$, and high reflectors $(n=16$; $[11-20] ; M=13.31, S D=2.75), p s>.26$.

fMRI data In order to compare the present paradigm with previously reported data on go/no-go tasks, we computed the whole-brain contrast correct no-go $>$ null events. In line with this literature, we found significant clusters of brain activation in the bilateral IFG and insular cortex and in the striatum (see Table 3).

We wanted to explore brain regions that show a difference during inhibiting negative, as compared with inhibiting positive, information. To this end, we contrasted trials on which participants had to disengage from emotionally negative information (happy/no-go trials) with trials on which participants had to disengage from emotionally positive information (sad/no-go trials). Most important, we were interested in brooding-related differences in activation during the inhibition of negative, as compared with positive, information. When interindividual brooding scores were correlated with the whole-brain contrast (happy/no-go $>\mathrm{sad} /$ no-go), we found a positive correlation within the right DLPFC (BA 46; MNI coordinates: 39, 35, 32; see Fig. 2) when applying small volume cluster correction at $p<.01$ within a mask comprising the frontal cortex on the basis of our a priori hypothesis. The scatterplot of the extracted percentage of signal changes is shown in order to rule out the possibility of the correlation's being driven by outliers. When including the covariate BDI in the model, the cluster in the right DLPFC was still present (SVC in the frontal cortex, cluster level corrected $p<.05$ ).

A similar correlation between activation level in the right DLPFC and BDI score was not significant, $r(34)=.21, p=.23$.

In order to relate our fMRI results to the behavioral performance, we correlated the whole-brain data of the contrast inhibiting-negative-inhibiting-positive (happy/nogo $<$ sad/no-go) with the behavioral difference in accuracy in inhibiting-negative-inhibiting-positive information. In line with our prefrontal predictions, we found a negative correlation in the right frontal cortex in the IFG, but not in the DLPFC (IFG, BA 45; MNI coordinates: 42, 42, 0; when applying small volume correction in the bilateral frontal 
Table 3 MNI coordinates of the contrast correct no-go $>$ null event (FWE corrected $p<.05$ )

\begin{tabular}{lcccc}
\hline Area & BA & Peak Coordinates (MNI) & Z Score & Voxel Extent \\
\hline Right visual cortex & 19 & $42,-60,-18$ & 7.58 & 788 \\
Right IFG/insula & $45 / 13$ & $39,18,0$ & 7.29 & 186 \\
Left IFG/insula & $45 / 13$ & $-35,14,4$ & 7.26 & 159 \\
Rostral cingulate zone & 24 & $0,21,39$ & 7.20 & 231 \\
Left thalamus & & $-11,-18,11$ & 6.30 & 79 \\
Right supplementary motor cortex & 6 & $14,11,67$ & 5.80 & 33 \\
Right supramarginal gyrus & 40 & $60,-42,25$ & 5.48 & 10 \\
Left striatum & & $-14,4,7$ & 5.29 & 8 \\
\hline
\end{tabular}

cortex, the region survives cluster correction at $p<.01$; see Fig. 3). This finding shows that the smaller the accuracy difference between inhibiting negative and positive information, the higher the activity in the IFG.

According to a median split of the right DLPFC activation, (nearly) significant differences can be observed in the number of correct inhibitions of negative information, $t(32)=1.99, p=.056$, and the number of correct inhibitions of happy information, $t(32)=2.47, p=.019$. These findings indicate that people with higher DLPFC activity more successfully inhibit sad and happy information. These findings are in line with DLPFC activation's being related to increased attentional control.

Ratings Rating data were entered into two mixed ANOVAs with within-subjects factors emotion (happy, sad) $\times$ group (low brooders, high brooders). For the first ANOVA, the dependent variable was the rating of valance (a higher rating indicating more positive valence). The results revealed a main effect of emotion, $F(1,32)=289.30, p<$ $.001, \eta^{2}=.90$, due to more negative ratings for negative faces $(M=3.09, S D=0.84)$, relative to positive faces, $(M=$ 6.93, $S D=0.62), t(33)=17.03, p<.001$. The other main effect of group and the interaction effects were not significant, $F \mathrm{~s}<1.29$. For the second ANOVA, the dependent variable was the rating of arousal (higher ratings indicating more arousal). The results revealed no main effects and a nonsignificant interaction effect, $F_{\mathrm{S}}<1.13$. Valence and arousal ratings were not correlated for happy and sad faces, $p \mathrm{~s}>.18$. Moreover, independent $t$ tests indicated that high and low brooders were not different in ratings of valance and arousal (for happy faces, $p \mathrm{~s}>.47$, and for sad faces, $p \mathrm{~s}>.23$ ). These findings suggest that behavioral and neural differences between high and low brooders are not based on interindividual differences in arousal of valence ratings.

\section{Discussion}

In the present study, event-related fMRI was employed to uncover the neural underpinnings of emotional disengage-

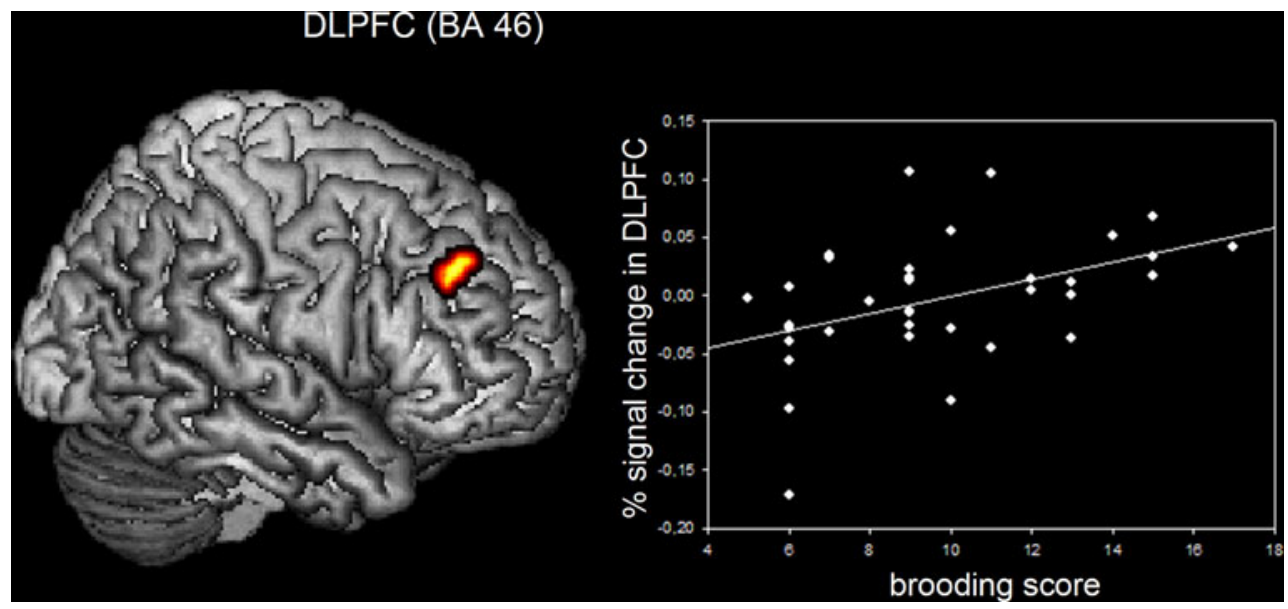

Fig. 2 Brain activity of the contrast correct inhibiting sad versus correct inhibiting happy showing a positive correlation with brooding. Activation map averaged thresholded with $p<.001$, cluster $>10$ voxels mapped onto a T1-weighted MNI single-subject template (colin27). On the right, a scatterplot of percentage of signal changes in the dorsolateral prefrontal cortex (DLPFC) and brooding scores 
Fig. 3 Brain activity of the contrast inhibiting sad versus inhibiting happy correctly showing a negative correlation with the accuracy difference of correct inhibition of sad versus happy.

Activation map averaged thresholded with $p<.001$, cluster $>10$ voxels mapped onto a T1weighted MNI single-subject template (colin27). On the right, a scatterplot of percentage of signal changes in the right inferior frontal gyrus (IFG) and accuracy differences

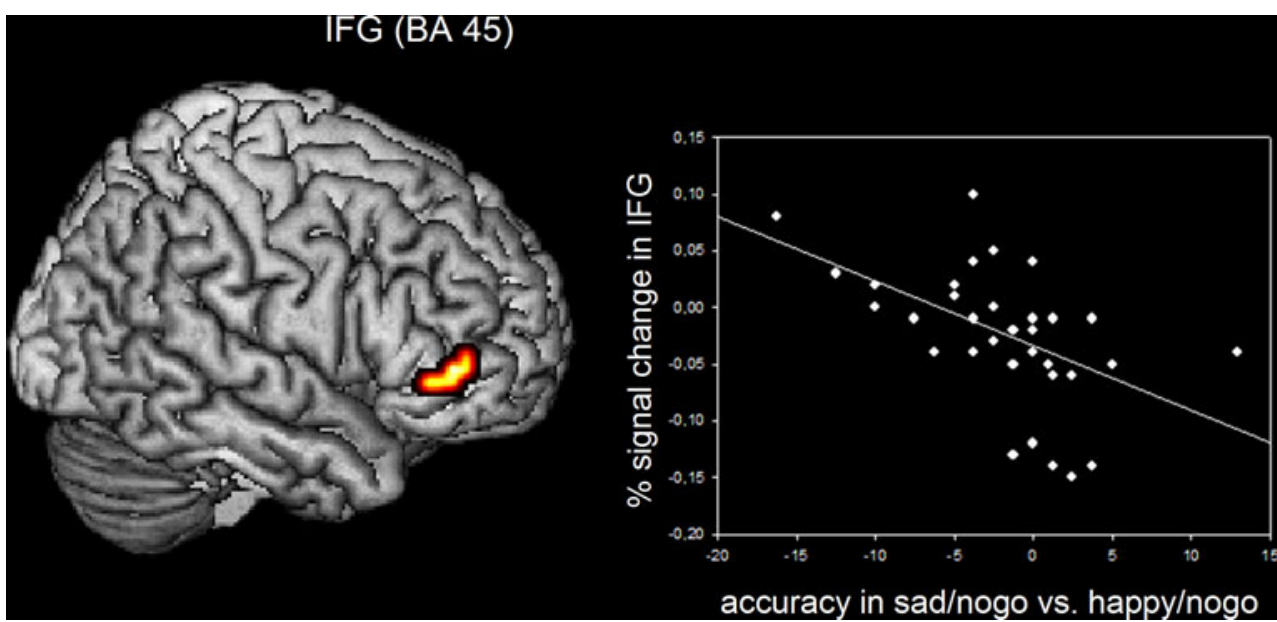

ment in relation to depressive brooding. A sample of healthy, never depressed individuals was selected to investigate whether attentional control processes were related to interindividual differences in depressive brooding. Thirty-four participants performed an emotional go/nogo task with emotionally positive and negative information.

Initially, behavioral data were not correlated with brooding scores across the entire group. Only for high brooders, however, did behavioral data reveal a negative correlation between interindividual differences in brooding tendencies and the difficulty of disengaging from negative information. More specifically, higher brooding tendencies were associated with more errors when disengaging from negative, relative to positive, information. In low brooders, we observed no correlation between accuracy rates and brooding scores. We propose that the absence of findings in low brooders is due to the fact that the range of interindividual differences in brooding tendencies was larger in high brooders.

In terms of neural activity, in line with robust neural findings using a go/no-go paradigm, the IFG and striatum were activated during the inhibition of prepotent responses (Aron \& Poldrack, 2006; Aron et al., 2004). On the basis of prior literature on interindividual differences in brooding scores, we were interested in the contrast between the inhibition of negative and positive information, which allowed us to focus on differential emotional disengagement. On the basis of a whole-brain analysis, we found that self-reported brooding scores correlated positively with activation in the right DLPFC (BA 46) during disengagement from negative information. In other words, individuals with high brooding scores showed more activation in the right DLPFC (BA 46) when disengaging from negative information. DLPFC regions have been shown to be involved in (1) setting a top-down attentional set oriented toward task-relevant information and away from task- irrelevant information (MacDonald et al., 2000; Milham, Banich, \& Barada 2003), (2) cognitive control for emotional information - for example, when distracting emotional information needs to be ignored during an emotional Stroop task (Etkin, Egner, Peraza, Kandel, \& Hirsch 2006; for a review, see Ochsner \& Gross, 2005)—and (3) disengagement from negative information (De Raedt et al., 2010). Moreover, previous literature has shown that negative mood states increase signal strength in the right DLPFC (for a review, see Banich et al., 2009; Davidson, 1998).

Therefore, increased activation in the right DLPFC during disengaging from negative information might be based on enhanced attentional control in the processing of emotionally negative information. This ability to control the processing of emotional material appears to be closely related to the ability to regulate how emotions are experienced and expressed. Indeed, the DLPFC has been implicated in different forms of emotion regulation, such as suppression and reappraisal (Ochsner \& Gross, 2005). Therefore, high brooders may have more need to regulate emotional processing in order to disengage successfully from negative information. Thus, they may be able to regulate their brooding processes to maintain healthy emotional functioning. All participants were healthy, never depressed patients who were not dysphoric (BDI scores were relatively low: $M=5.52, S D=3.95$ ). It may be that individuals with elevated BDI scores fail to regulate their brooding processes in order to disengage from negative information, a deficit that might be related to the development and maintenance of depressive episodes. Although it has been shown that depressed patients demonstrate an inability to disengage from negative information and are less able to use adaptive emotion regulation strategies (Joormann, 2010), further neuroimaging research is needed to relate this emotion regulation inability to brooding tendencies. 
Moreover, additional research is needed to explore the specific nature of the behavioral correlation in the right IFG, as compared with the depressive brooding correlation in the DLPFC. On the one hand, the right IFG is implicated in the difference in accuracy between inhibiting negative and inhibiting positive information, which appears to suggest a correlations with motor inhibition processes. The better the performance on inhibiting positive (sad/no-go) information, relative to inhibiting negative (happy/no-go) information, the stronger the activation in the right IFG during sad/no-go, as compared with happy/no-go. This region in the right IFG is not correlated with brooding. This could indicate that the right IFG is specifically related to the inhibition of behavioral motor output (e.g., action monitoring), an observation that has been frequently discussed in the literature (e.g., Aron et al., 2004). On the other hand, when exploring which brain regions are specifically associated with depressive brooding during the inhibition of negative content, as compared with happy content, a correlation within the DLPFC was obtained. This finding could suggest that depressive brooding is not necessarily directly related to the behavioral output (accuracy rates) but, rather, is related to the cognitive processing of the negative stimulus material. This is in line with the finding of a study in depressed patients that showed that activation of the DLPFC by means of repetitive transcranial magnetic stimulation was related to an enhanced inhibition of negative material (Leyman, De Raedt, Vanderhasselt, \& Baeken 2011). The latter inhibition processes were measured using the negative affective priming task, which provides a measure of inhibition that is unrelated to response inhibition (Joormann, 2004). Similar to the present findings, the DLPFC was more strongly implicated in the inhibition of negative, as compared with positive, content but was unrelated to the process of response selection (e.g., higher level cognitions). Thus, it appears that the association of the IFG with behavioral performance argues for a link with response inhibition processes, whereas the association between the DLPFC and brooding argues in favor of an association with the disengagement (i.e., cognitive inhibition) from negative thought content. However, more research is needed to explore the interplay between the IFG and DLPFC and behavioral measures.

A limited generalizability of the behavioral results should be noted as a first limitation of this study. Although not significantly different from each other, it is puzzling that the absolute accuracy rates of the high brooders were somewhat higher than those of the low brooders. As for the correlations, brooding scores were correlated with the emotional disengagement score only in the high brooding group $(n=18)$, but not across all participants $(n=34)$ (see Fig. 3). This dichotomization of a quantitative measure is usually discouraged because it reduces the power or, on the other hand, overestimates the significance of the effect observed (MacCallum, Zhang, Preacher, \& Rucker 2002). But the distribution of the lower brooding scores was rather narrow, whereas the other part of the brooding scores demonstrated a comparably broad distribution. On the basis of a median split, the range and standard deviation in the low brooding group $(n=19 ;[5-9] ; M=7.22, S D=1.44)$ was much smaller, as compared with the high brooding group $(n=15 ;[10-17] ; M=12.50, S D=2.28)$. This may explain why we observed correlations with the behavioral data only in the high brooders, not across all participants. However, more research is needed to clarify why the association between depressive brooding and accuracy rates was not present across the entire sample. A second limitation of this study is that there was no neutral condition in the emotional go/no-go task. It might therefore be that our findings are based on responding to the valence of go trials and that the no-go contrast was confounded with the valence of the face. We chose not to use neutral faces because, on the basis of a pilot study, accuracy rates for neutral faces were relatively low. We think that this was due to the fact that neutral faces can easily be evaluated as being sad. This would imply that a neutral face is not necessarily a neutral condition. Moreover, the emotional evaluation of neutral faces has been associated with increased amygdala activation (Brotman et al., 2010).

In conclusion, at the behavioral level, high brooders were less capable (i.e., made more errors) of disengaging from negative information than were low brooders. In addition, high brooders evoked more right DLPFC activation in successfully disengaging from the negative. Ultimately, our data appear to suggest that high brooders who are clinically healthy compensate for their difficulty in inhibiting negative information by recruiting more attentional control, enabling them to disengage successfully at a behavioral level. This mechanism might be based on an increased use of emotion regulation strategies. These findings are specific to healthy, nondysphoric, and never depressed high brooders. It is likely that these emotion regulation mechanisms would not function as effectively in a depressive brooder who is dysphoric. Further research is needed in depressed samples to investigate the neural correlates of attentional control when disengaging from negative information.

Acknowledgement MAV (FWO08/PDO/168) and SK are postdoctoral fellows of the Research Foundation Flanders (FWO). The authors are grateful for the help of Jerome Tullo with scientific interpretation and the drafting of this article. The authors declare no conflicts of interest. 


\section{References}

Aron, A. R., \& Poldrack, R. A. (2006). Cortical and subcortical contributions to stop signal response inhibition: Role of the subthalamic nucleus. The Journal of Neuroscience, 26, 24242433. doi:10.1523/JNEUROSCI.4682-05.2006.

Aron, A. R., Robbins, T. W., \& Poldrack, R. A. (2004). Inhibition and the right inferior frontal cortex. Trends in Cognitive Sciences, 8, 170-177. doi:10.1016/j.tics.2004.02.010.

Banich, M. T., Mackiewicz, K. L., Depue, B. E., Whitmer, A. J., Miller, G. A., \& Heller, W. (2009). Cognitive control mechanisms, emotion and memory: A neural perspective with implications for psychopathology. Neuroscience and Biobehavioral Reviews, 33, 613-630. doi:10.1016/j.neubiorev.2008.09.010.

Beck, A. T., Steer, R. A., \& Brown, G. K. (1996). Manual for the Beck depression inventory (2nd ed.). San Antonio: The Psychological Corporation.

Berman, M. G., Nee, D. E., Casement, M., Kim, H. S., Deldin, P., Kross, E., . . Jonides, J. (2011). Neural and behavioral effects of interference resolution in depression and rumination. Cognitive Affective, \& Behavioral Neuroscience. doi:10.3758/s13415-0100014-x

Berman, M. G., Peltier, S., Nee, D. E., Kross, E., Deldin, J. D., \& Jonides, J. (2011). Depression, rumination and the default network. Social Cognitive and Affective Neuroscience. doi:10.1093/scan/nsq080

Brett, M., Anton, J.-L., Valabregue, R., \& Poline, J.-B. (2002, June). Region of interest analysis using an SPM toolbox. Paper presented at the 8th International Conference on Functional Mapping of the Human Brain, Sendai, Japan.

Brotman, M. A., Rich, B. A., Guyer, A. E., Lunsford, J. R., Horsey, S. E., Reising, M. M., et al. (2010). Amygdala activation during emotion processing of neutral faces in children with severe mood dysregulation versus ADHD or bipolar disorder. The American Journal of Psychiatry, 167, 61-69. doi:10.1176/appi.ajp.2009.09010043.

Davidson, R. J. (1998). Affective style and affective disorders: Perspectives from affective neuroscience. Cognition and Emotion, 12, 307-320.

De Lissnyder, E., Koster, E. H. W., Derakshan, N., \& De Raedt, R. (2010). The association between depressive symptoms and executive control impairments in response to emotional and non-emotional information. Cognition \& Emotion, 24, 264-280. doi:10.1080/02699930903378354.

De Raedt, R., \& Koster, E. H. W. (2010). Understanding vulnerability for depression from a cognitive neuroscience perspective: A reappraisal of attentional factors and a new conceptual framework. Cognitive, Affective \& Behavioral Neuroscience, 10, 50-70. doi:10.3758/ cabn.10.1.50.

De Raedt, R., Leyman, L., Baeken, C., Van Schuerbeek, P., Luypaert, R., Vanderhasselt, M. A., et al. (2010). Neurocognitive effects of Hf-rTMS over the dorsolateral prefrontal cortex on the attentional processing of emotional information in healthy women: An event-related fMRI study. Biological Psychology, 85, 487-495. doi:10.1016/j.biopsycho.2010.09.015.

Etkin, A., Egner, T., Peraza, D. M., Kandel, E. R., \& Hirsch, J. (2006). Resolving emotional conflict: A role for the rostral anterior cingulate cortex in modulating activity in the amygdala. Neuron, 51, 871-882. doi:10.1016/j.neuron.2006.07.029.

Goeleven, E., De Raedt, R., Leyman, L., \& Verschuere, B. (2008). The Karolinska Directed Emotional Faces: A validation study. Cognition \& Emotion, 22, 1094-1118. doi:10.1080/ 02699930701626582.

Gross, J. J., \& John, O. P. (2003). Individual differences in two emotion regulation processes: Implications for affect, relationships, and well-being. Journal of Personality and Social Psychology, 85, 348-362. doi:10.1037/0022-3514.85.2.348.
Hertel, P. T. (2007). Impairments in inhibition or cognitive control in psychological disorders. Applied \& Preventive Psychology, 12, 149-153. doi:10.1016/j.appsy.2007.09.006.

Joormann, J. (2004). Attentional bias in dysphoria: The role of inhibitory processes. Cognition \& Emotion, 18, 125-147.

Joormann, J. (2010). Cognitive inhibition and emotion regulation in depression. Current Directions in Psychological Science, 19, 161-166. doi:10.1177/0963721410370293.

Joormann, J., Dkane, M., \& Gotlib, I. H. (2006). Adaptive and maladaptive components of rumination? Diagnostic specificity and relation to depressive biases. Behavior Therapy, 37, 269280.

Koster, E. H. W., De Lissnyder, E., Derakshan, N., \& De Raedt, R. (2010). Understanding depressive rumination from an affective neuroscience perspective: The impaired disengagement hypothesis. Clinical Psychology Review, 31, 138-145.

Leyman, L., De Raedt, R., Vanderhasselt, M. A., \& Baeken, C. (2009). Influence of high-frequency repetitive transcranial magnetic stimulation over the dorsolateral prefrontal cortex on the inhibition of emotional information in healthy volunteers. Psychological Medicine, 39, 1019-1028. doi:10.1017/s0033291708004431.

Leyman, L., De Raedt, R., Vanderhasselt, M. A., \& Baeken, C. (2011). Effects of repetitive transcranial magnetic stimulation of the dorsolateral prefrontal cortex on the attentional processing of emotional information in major depression: A pilot study. Psychiatry Research, 185, 102-107.

Lundqvist, D., Flykt, A., \& Öhman, A. (1998). The Karolinska Directed Emotional Faces (KDEF). Stockholm: Karolinska Hospital, Department of Neurosciencesl.

MacCallum, R. C., Zhang, S. B., Preacher, K. J., \& Rucker, D. D. (2002). On the practice of dichotomization of quantitative variables. Psychological Methods, 7, 19-40. doi:10.1037//1082989x.7.1.19.

MacDonald, A. W., Cohen, J. D., Stenger, V. A., \& Carter, C. S. (2000). Dissociating the role of the dorsolateral prefrontal and anterior cingulate cortex in cognitive control. Science, 288, $1835-1838$.

Maldjian, J. A., Laurienti, P. J., Burdette, J. B., \& Kraft, R. A. (2003). An automated method for neuroanatomic and cytoarchitectonic atlas-based interrogation of fMRI data sets. Neuroimage, 19, 1233-1239. doi:10.1016/S1053-8119(03)00169-1.

Milham, M. P., Banich, M. T., \& Barada, V. (2003). Competition for priority in processing increases prefrontal cortex's involvement in top-down control: An event-related fMRI study of the Stroop task. Cognitive Brain Research, 17, 212-222. doi:10.1016/ s0926-6410(03)00108-3.

Nolan, S. A., Roberts, J. E., \& Gotlib, I. H. (1998). Neuroticism and ruminative response style as predictors of change in depressive symptomatology. Cognitive Therapy and Research, 22, 445-455.

Nolen-Hoeksema, S. (2000). The role of rumination in depressive disorders and mixed anxiety/depressive symptoms. Journal of Abnormal Psychology, 109, 504-511.

Nolen-Hoeksema, S., \& Morrow, J. (1991). A prospective study of depression and posttraumatic stress symptoms after a natural disaster: The 1989 Loma Prieta earthquake. Journal of Personality and Social Psychology, 61, 115-121.

Ochsner, K. N., \& Gross, J. J. (2005). The cognitive control of emotion. Trends in Cognitive Sciences, 9, 242-249. doi:10.1016/j. tics.2005.03.010.

Raes, F., \& Hermans, D. (2007). The revised version of the Dutch Ruminative Response Scale. Unpublished instrument.

Ray, R. D., Ochsner, K. N., Cooper, J. C., Robertson, E. R., Gabrieli, J. D. E., \& Gross, J. J. (2005). Individual differences in trait rumination and the neural systems supporting cognitive reappraisal. Cognitive, Affective \& Behavioral Neuroscience, 5, 156168. 
Roberts, J. E., Gilboa, E., \& Gotlib, I. H. (1998). Ruminative response style and vulnerability to episodes of dysphoria: Gender, neuroticism, and episode duration. Cognitive Therapy and Research, 22, 401-423.

Robinson, M. S., \& Alloy, L. B. (2003). Negative cognitive styles and stress-reactive rumination interact to predict depression: A prospective study. Cognitive Therapy and Research, 27, 275-291.

Schoofs, H., Hermans, D., \& Raes, F. (2011). Brooding and reflection as subtypes of rumination: Evidence from confirmatory factor analysis in nonclinical samples using the Dutch Ruminative Response Scale. Journal of Psychopathology and Behavioral Assessment.

Schulz, K. P., Fan, J., Magidina, O., Marks, D. J., Hahn, B., \& Halperin, J. M. (2007). Does the emotional go/no-go task really measure behavioral inhibition? Convergence with measures on a non-emotional analog. Archives of Clinical Neuropsychology, 22 (2), 151-160. doi:10.1016/j.acn.2006.12.001.

Siegle, G. J., Steinhauer, S. R., Thase, M. E., Stenger, V. A., \& Carter, C. S. (2002). Can't shake that feeling: Event-related fMRI assessment of sustained amygdala activity in response to emotional information in depressed individuals. Biological Psychiatry, 51, 693-707.

Treynor, W., Gonzalez, R., \& Nolen-Hoeksema, S. (2003). Rumination reconsidered: A psychometric analysis. Cognitive Therapy and Research, 27, 247-259.

Van der Does, A. J. W. (2002). De Nederlandse versie van de Beck depression inventory-Tweede Editie [The Dutch version of the Beck depression inventory-Second edition]. Lisse: Swets \& Zeitlinger.

Vanderhasselt, M. A., De Raedt, R., \& Baeken, C. (2009). Dorsolateral prefrontal cortex and Stroop performance: Tackling the lateralization. Psychonomic Bulletin \& Review, 16, 609-612. doi:10.3758/pbr.16.3.609.

Vanderhasselt, M. A., De Raedt, R., Baeken, C., Leyman, L., Clerinx, P., \& D'Haenen, H. (2007). The influence of rTMS over the right dorsolateral prefrontal cortex on top-down attentional processes. Brain Research, 1137, 111-116. doi:10.1016/j.brainres.2006.12.050.

Whitmer, A. J., \& Banich, M. T. (2007). Inhibition versus switching deficits in different forms of rumination. Psychological Science, $18,546-553$. 